\title{
ON THE NOTION OF PHASE IN MECHANICS
}

\author{
Maurice A de Gosson \\ BTH-Karlskrona \\ SE-371 79 Karlskrona
}

October 24, 2018

\begin{abstract}
The notion of phase plays an essential role in both semiclassical and quantum mechanics. But what is exactly a phase, and how does it change with time? It turns out that the most universal definition of a phase can be given in terms of Lagrangian manifolds by exploiting the properties of the Poincaré-Cartan form. Such a phase is defined, not in configuration space, but rather in phase space and is thus insensitive to the appearance of caustics. Surprisingly enough this approach allows us to recover the Heisenberg-Weyl formalism without invoking commutation relations for observables.
\end{abstract}

\section{Contents}

\begin{tabular}{lll}
\hline 1 & Introduction & 2
\end{tabular}

2 Lagrangian Manifolds in Mechanics 5

3 The Phase of a Lagrangian Submanifold 6

4 The Local Expression of the Phase 10

5 Svmplectic Frames and Lagrangian Phases 11

6 Hamiltonian Motions and Phase 14

$\begin{array}{lll}7 & \text { Phase and Heisenberg-Wevl Operators } & 18\end{array}$ 


\section{Introduction}

What is a phase? A common conception is that it is something like an angle; but this does of course not tell us very much concretely. Let us look up the word "phase" in the Webster". We find there that "... [a phase is] the stage of progress in a regularly recurring motion or a cyclic progress (as a wave or vibration) in relation to a reference point." The last few words really go straight to the point: the vocation of a phase is to describe a variation -it has no absolute meaning by itself. So what would then a good definition of the variation of "phase" be for a mechanical system? Consider a Hamiltonian system (in $n$ degrees of freedom) with Hamiltonian $H=H(x, p, t)$; here $x=\left(x_{1}, \ldots, x_{n}\right), p=\left(p_{1}, \ldots, p_{n}\right)$. We will define the variation of the phase of that system when it evolves from a state $z^{\prime}=\left(x^{\prime}, p^{\prime}\right)$ at time $t^{\prime}$ to a state $z=(x, p)$ at time $t$ by the formula

$$
\Delta \Phi=\int_{z^{\prime}, t^{\prime}}^{z, t} p \mathrm{~d} x-H \mathrm{~d} t
$$

where the integration is performed along the arc joining $\left(z^{\prime}, t^{\prime}\right)$ to $(z, t)$ in time-dependent phase space, and determined by the Hamilton equations for $H$.

So far, so good. But again: what is then "the" phase of that system? A clue is given by Hamilton-Jacobi's equation with initial datum

$$
\frac{\partial \Phi}{\partial t}+H\left(x, \nabla_{x} \Phi\right)=0, \quad \Phi\left(x, t^{\prime}\right)=\Phi^{\prime}(x) .
$$

Assume that $H$ is of the classical type "kinetic energy + smooth potential"; then the solution of the problem (2) always exists (and is unique) if $\left|t-t^{\prime}\right|$ is sufficiently small. This solution $\Phi=\Phi(x, t)$ is obtained as follows. Let us denote by $\left(f_{t, t^{\prime}}^{H}\right)$ the time-dependent flow determined by $H$ and consider the graph $\mathbb{V}^{\prime}$ of the function $p^{\prime}=\nabla_{x} \Phi^{\prime}\left(x^{\prime}\right)$. For small values of $\left|t-t^{\prime}\right|$ the image $\mathbb{V}=f_{t, t^{\prime}}^{H}\left(\mathbb{V}^{\prime}\right)$ will still project diffeomorphically on configuration space and hence still be a graph; the coordinate $x$ being given let $p$ be the unique momentum vector such that $z=(x, p) \in \mathbb{V}$ and define $z^{\prime}=\left(x^{\prime}, p^{\prime}\right) \in \mathbb{V}^{\prime}$ by $z=f_{t, t^{\prime}}^{H}\left(z^{\prime}\right)$; then the difference $\Phi(x, t)-\Phi^{\prime}\left(x^{\prime}\right)$ is the quantity

$$
\Delta \Phi\left(x, x^{\prime}\right)=\int_{z^{\prime}, t^{\prime}}^{z, t} p \mathrm{~d} x-H \mathrm{~d} t .
$$

\footnotetext{
${ }^{1}$ Webster New Encyclopedia, 1994 edition.
} 
and we can take the formula

$$
\Phi(x, t)=\Phi^{\prime}\left(x^{\prime}\right)+\int_{z^{\prime}, t^{\prime}}^{z, t} p \mathrm{~d} x-H \mathrm{~d} t
$$

as a definition of the phase of the Hamiltonian system. Such a choice is quite correct, and very much in the spirit of Hamilton-Jacobi theory. It is however too restrictive, because the definition of $\Phi(x, t)$ heavily relies on the fat that we were able to define a point $x^{\prime}$ on the initial graph $\mathbb{V}^{\prime}$ via the formula $(x, p)=f_{t, t^{\prime}}^{H}\left(x^{\prime}, p^{\prime}\right)$. This is only possible if $\mathbb{V}=f_{t, t^{\prime}}^{H}\left(\mathbb{V}^{\prime}\right)$ is itself is a graph, and this is in general no longer the case when $\left|t-t^{\prime}\right|$ becomes too large: for a given $x$ there will perhaps be several points $\left(x, p_{1}\right),\left(x, p_{2}\right), \ldots$, of $\mathbb{V}$ having the same position coordinate due to the "bending" of $\mathbb{V}^{\prime}$ by the flow as time elapses, and formula (3) will no longer make sense (to use an older terminology, the phase becomes "multi-valued"). This is the usual problem to which one is confronted to in Hamilton-Jacobi theory, and is also, by the way, one of the reasons for which the WKB method breaks down for large times: the semiclassical solutions to Schrödinger's equation one wants to define on the set $\mathbb{V}=f_{t, t^{\prime}}^{H}\left(\mathbb{V}^{\prime}\right)$ blow up because of the appearance of "caustics" related to that bending. In semiclassical mechanics, the remedy to this situation is well-known: one renounces to the usual solutions of Hamilton-Jacobi's equation (2) and one considers the manifolds $\mathbb{V}$ themselves -whether they are graphs, or not- as generalized solutions: this is the phase space approach to semiclassical mechanics inaugurated by Keller, and further developed by Maslov [15], Maslov and Fedoriuk [16, Leray [12, and many others. Now, these manifolds are not arbitrary; they are Lagrangian submanifolds of phase space. These special manifolds can be thought as generalizations of the usual invariant tori of Liouville integrable systems of Hamiltonian mechanics, but there use is certainly not limited to this venerable topic: Lagrangian manifolds have a life of their own, and intervene in various fields. Even if one doesn't have to take Weinstein's [19] creed "everything is a Lagrangian manifold!" quite at face value, it is however true that Lagrangian manifolds can be associated in a very natural way both to classical and quantum systems. (We will discuss this in some detail in Section 2] in any case the solution of Cauchy's problem for Hamilton-Jacobi's equation anyway involves de facto a Lagrangian manifold, whether the system is Liouville integrable or not.) The situation is even more clear-cut in quantum mechanics: to every quantum system whose evolution is governed by Schrödinger's equation

$$
i \hbar \frac{\partial \Psi}{\partial t}=\hat{H} \Psi
$$


one can associate a canonical Lagrangian manifold: writing the wavefunction in polar form $\Psi=\exp (i \Phi / \hbar)$ the graph $p=\nabla_{x} \Phi(x, t)$ of the phase at time $t$ is a Lagrangian manifold. (We have used this fact in [6] to show how this can be used to understand Schrödinger's equation in the framework of the Hamilton-Jacobi formalism.)

An essential tool for the study of the time-evolution of the phase of a Lagrangian manifold under the action of Hamiltonian flows is the PoincaréCartan form $p \mathrm{~d} x-H \mathrm{~d} t$. Its importance comes from the fact that it is a (relative) integral invariant. Strangely enough, this property is often mentioned in both the mathematical and physical literature, but seldom fully exploited. Admittedly, the approach "Lagrangian manifolds + PoincaréCartan invariant" is certainly not new; for instance Weinstein [20] has used it to study the global properties of paths of Lagrangian manifold subject to an "isodrastic" (that is, action-preserving) deformation; due to the heavy use of intrinsic differential geometry Weinstein's paper is however not easily accessible to a physical audience. On the other hand many of the results contained in Section 2 can be found in an elusive or fragmentary form elsewhere (e.g. [5, 12]). In Section 7 we show how the properties of the phase allows us to recover the Heisenberg-Weyl operator formalism familiar from semiclassical mechanics.

This article is relatively self-contained: the proofs are complete (even if concise), and we have found it useful to shortly review the necessary topics from symplectic geometry and Hamiltonian mechanics (the invariance property of the Poincaré-Cartan form is one example); we refer the reader to the classical treatises [2, 18, 1, 13] (cited in increasing order of mathematical difficulty) for the notions of differential geometry that we will use.

Notations. The phase space $\mathbb{R}_{z}^{2 n}=\mathbb{R}_{x}^{n} \times \mathbb{R}_{p}^{n}$ is equipped with the standard symplectic form $\sigma$ :

$$
\sigma\left(z, z^{\prime}\right)=p x^{\prime}-p^{\prime} x=\sum_{j=1}^{n} p_{j} x_{j}^{\prime}-p_{j}^{\prime} x_{j}
$$

if $z=(x, p), z^{\prime}=\left(x^{\prime}, p^{\prime}\right)$; in differential notation:

$$
\sigma=\mathrm{d} p \wedge \mathrm{d} x=\sum_{j=1}^{n} \mathrm{~d} p_{j} \wedge \mathrm{d} x_{j} .
$$

A Lagrangian plane is a $n$-dimensional linear subspace $\ell$ of $\mathbb{R}_{z}^{2 n}$ such that the symplectic form $\sigma$ vanishes on every pair of vectors of $\ell$ :

$$
z, z^{\prime} \in \ell \Longrightarrow \sigma\left(z, z^{\prime}\right)=0 \text {. }
$$


Equivalent definitions are: $(*)$ a Lagrangian plane is the image of configuration space $\mathbb{R}_{x}^{n}$ (or momentum space $\mathbb{R}_{p}^{n}$ ) by a linear symplectic transformation (i.e. a symplectic matrix); (**) A $n$-plane with equation $A x+B p=0$ is Lagrangian if and only if $A^{T} B=B A^{T}$.

In all what follows the letter $\mathbb{V}$ will denote a connected (but not necessarily compact) Lagrangian submanifold of the phase space $\mathbb{R}_{z}^{2 n}$, that is:

- $\mathbb{V}$ has dimension $n$ as a manifold;

- the tangent space $\ell(z)=T_{z} \mathbb{V}$ at every point $z$ of $\mathbb{V}$ is a Lagrangian plane.

\section{Lagrangian Manifolds in Mechanics}

A basic (but not generic) example of Lagrangian manifold is the following: let $\Phi=\Phi(x)$ be a smooth function defined on some open domain in configuration space. Then

$$
\mathbb{V}: p=\nabla_{x} \Phi(x)
$$

is a Lagrangian manifold (sometimes called "exact Lagrangian manifold"). The image of a Lagrangian manifold $\mathbb{V}$ by a symplectic diffeomorphism $f$ is again a Lagrangian manifold: $f(\mathbb{V})$ is a manifold, and the tangent mapping $\mathrm{d} f\left(z_{0}\right)$ is an isomorphism of $\ell\left(z_{0}\right)=T_{z_{0}} \mathbb{V}$ on $\ell\left(f\left(z_{0}\right)\right)=T_{f\left(z_{0}\right)} f(\mathbb{V})$; that isomorphism is symplectic hence $\ell\left(f\left(z_{0}\right)\right.$ is a Lagrangian plane. Observe that a Lagrangian plane is a Lagrangian manifold in it own right, and so is the image by a Lagrangian plane by a symplectic diffeomorphism.

Let us begin by making the following pedestrian -but important- remark. Suppose that we have a system of $N$ point-like particles at some time, say $t=0$, and that we know all the positions and momenta of these particles; that system is thus identified with a point $z=(x, p)$ in phase space. We can always find a Lagrangian manifold (in fact, infinitely many) carrying this point $z$. The easiest example is obtained by choosing numbers $a_{1}, \ldots, a_{n}$ such that $p_{j}=a_{j} x_{j}$; denoting by $M$ the diagonal matrix with diagonal entries $a_{j}$, the linear space $\ell: p=M x$ is a Lagrangian plane (and hence, a fortiori, a Lagrangian manifold).

We can even do better: assume that the system of $N$ particles is Hamiltonian with Hamiltonian $H$, and let $E$ be the energy of the system. Consider a solution $\Phi=\Phi(x)$ of the reduced Hamilton-Jacobi equation

$$
H\left(x, \nabla_{x} \Phi\right)=E .
$$


The manifold $\mathbb{V}: p=\nabla_{x} \Phi(x)$ is Lagrangian and the energy of $H$ is constant on it; it thus lies on the energy shell $\Sigma_{E}: H(z)=E$. We thus see that independently of any integrability condition one can associate an exact Lagrangian manifold to every Hamiltonian system; that Lagrangian manifold can be interpreted as a set carrying a "cloud" of particles, in fact a statistical ensemble where the positions and momenta are in interrelated by the formula $p=\nabla_{x} \Phi(x)$. More generally, there is no need to assume ha there is such a correlation, and one can as well consider a Lagrangian manifold as a set representing a physical state. When one weights this manifold by a density (or rather a de Rham form, see [7, 8]) and thereafter imposing to it the Maslov(or $E B K$ ) quantum conditions, one obtains semiclassical mechanics.

It should be noted that Lagrangian (sub) manifolds actually play an ubiquitous role in physics. For instance, the role of "reciprocity laws" giving arise to such manifolds in thermodynamics ("Onsager relations"), thermostatics ("Maxwell relations"), and in electricity and electromagnetism is well-known. Tulczyjew and Oster actually view Lagrangian manifolds as the basic entities describing physical systems (see Abraham and Marsden [1], Ch. 5, for an extensive list of references and many examples).

\section{The Phase of a Lagrangian Submanifold}

et $\mathbb{V}$ be an exact Lagrangian manifold, defined by the equation $p=\nabla_{x} \Phi(x)$. Then $\varphi(z)=\Phi(x)$ is a phase of $\mathbb{V}$. This is obvious since

$$
\mathrm{d} \varphi(z)=\mathrm{d} \Phi(x)=p \mathrm{~d} x .
$$

We observe that the phase can be expressed as an integral:

$$
\varphi(z)=\Phi\left(x_{0}\right)+\int_{\gamma} \mathrm{d} \Phi(x)
$$

where $\gamma$ is any path in configuration space joining $x_{0}$ to $x$.

To see what a notion of phase could be for a Lagrangian manifold which is not a graph, let us begin with a simple example. We would like to define on the circle $S^{1}(R): x^{2}+p^{2}=R^{2}$ in the plane $\mathbb{R}_{z}^{2}$ a smooth function $\varphi$ whose differential $\mathrm{d} \varphi$ is the action form $p \mathrm{~d} x$. Passing to polar coordinates $x=R \cos \theta, p=R \sin \theta$ the condition $\mathrm{d} \varphi=p \mathrm{~d} x$ is

$$
\mathrm{d} \varphi(\theta)=-R^{2} \sin ^{2} \theta
$$

which, integrated, leads to

$$
\varphi(\theta)=\frac{R^{2}}{2}(\cos \theta \sin \theta-\theta) .
$$


Now, that function is not defined on the circle itself, because $\varphi(\theta+2 \pi)=$ $\varphi(\theta)-\pi R^{2} \neq \varphi(\theta)$. We can however view $\varphi(\theta)$ as defined on the universal covering of $S^{1}(R)$, identified with the real line $\mathbb{R}_{\theta}$, the projection $\pi: \mathbb{R}_{\theta} \longrightarrow$ $S^{1}(R)$ being given by $\pi(\theta)=(R \cos \theta, R \sin \theta)$.

Consider, more generally, a completely integrable system with Hamiltonian $H$, and $(\theta, I)=\left(\theta_{1}, \ldots, \theta_{n} ; I_{1}, \ldots, I_{n}\right)$ the corresponding angle-action variables. We have $H(x, p)=K(I)$ and the motion is given by

$$
\theta(t)=\theta(0)+\omega(I(0)) t \quad, \quad I(t)=I(0)
$$

where the frequency vector $\omega(I)=\left(\omega_{1}(I), \ldots, \omega_{n}(I)\right)$ is the gradient of $K$ : $\omega(I)=\nabla_{I} K(I)$. The motion takes place on the Lagrangian manifold $I(t)=$ $I(0)$. Topologically this manifold is identified with a product of $n$ unit circles, each lying in a plane of conjugate variables. Recalling that $\theta=\left(\theta_{1}, \ldots, \theta_{n}\right)$ the phase of $\mathbb{T}$ is thus

$$
\varphi(\theta)=\frac{1}{2} \sum_{j=1}^{n}\left(\cos \theta_{j} \sin \theta_{j}-\theta_{j}\right)
$$

in view of (4).

Consider now an arbitrary Lagrangian manifold $\mathbb{V}$, and choose a "base point" $\bar{z}=(\bar{x}, \bar{p})$ on $\mathbb{V}$; we denote by $\pi_{1}(\mathbb{V})$ the fundamental group $\pi_{1}(\mathbb{V}, \bar{z})$. Let us denote by $\check{\mathbb{V}}$ the set of all homotopy classes $\check{z}$ of paths $\gamma(\bar{z}, z)$ starting at $\bar{z}$ and ending at $z$, and by $\pi: \check{V} \longrightarrow \mathbb{V}$ the mapping which to $\check{z}$ associates the endpoint $z$ of any of its representatives $\gamma(\bar{z}, z)$. The set $\check{\mathbb{V}}$ can be equipped with a topology having the following properties: $(*) \check{\mathbb{V}}$ is simply connected; $(* *) \pi$ is a covering mapping: every $z \in \mathbb{V}$ has an open neighborhood $U$ such that $\pi^{-1}(U)$ is the disjoint union of a sequence of open sets $\check{U}_{1}, \check{U}_{2}, \ldots$ such that the restriction of $\pi$ to each of the $\check{U}_{j}$ is a diffeomorphism onto $U$. With that topology and projection, $\check{\mathbb{V}}$ is the universal covering of $\mathbb{V}$. Consider now the action form

$$
p \mathrm{~d} x=p_{1} \mathrm{~d} x_{1}+\cdots+p_{n} \mathrm{~d} x_{n}
$$

on $\mathbb{V}$; we can "pull-back" this form to $\check{\mathbb{V}}$ using the projection $\pi$, thus obtaining a one-form $\pi^{*}(p \mathrm{~d} x)$. Now

$$
\mathrm{d} \pi^{*}(p \mathrm{~d} x)=\pi^{*} \mathrm{~d}(p \mathrm{~d} x)=\pi^{*}(\mathrm{~d} p \wedge \mathrm{d} x)
$$

and $\mathrm{d} p \wedge \mathrm{d} x=\sigma$ is identically zero on $\mathbb{V}$, hence the form $\pi^{*}(p \mathrm{~d} x)$ is closed on $\check{\mathbb{V}}$. Since $\check{\mathbb{V}}$ is contractible $\pi^{*}(p \mathrm{~d} x)$ is an exact form on $\check{\mathbb{V}}$ in view of Poincaré's 
lemma and we can thus find infinitely many functions $\varphi: \check{\mathbb{V}} \longrightarrow \mathbb{R}$, all differing by a constant, such that $\mathrm{d} \varphi(\check{z})=\pi^{*}(p \mathrm{~d} x)$. Making a slight abuse of notation by identifying $p \mathrm{~d} x$ and its pull-back $\pi^{*}(p \mathrm{~d} x)$ we can summarize the discussion above as follows:

$$
\begin{aligned}
& \text { There exists a differentiable function } \varphi: \check{V} \longrightarrow R \text { such that } \\
& \qquad \mathrm{d} \varphi(\check{z})=p \mathrm{~d} x \text { if } \pi(\check{z})=z=(x, p) .
\end{aligned}
$$

We will call such a function a phase of $\mathbb{V}$, although $\varphi$ is in general defined on the universal covering $\check{\mathbb{V}}$. Notice that we can always fix one such phase by imposing a given value at some point of $\check{\mathbb{V}}$; for instance we can choose $\varphi(\bar{z})=0$ where $\bar{z}$ is identified with the (homotopy class of) the constant loop $\gamma(\bar{z}, z)$.

A straightforward example of phase one can associate to a system of particles represented by a phase space point $z$ is the following:

Example 1 Let $\ell: p=M x$ be a Lagrangian plane passing through $z$ (that such a plane always exists was discussed above). Choosing the origin as base point: $\bar{z}=0$ the phase is

$$
\phi(z)=\frac{1}{2} p \cdot x=\frac{1}{2} M x^{2}
$$

(where we have set $\left.M x^{2}=M x \cdot x\right)$.

Phases on Lagrangian manifolds can be explicitly constructed by integrating the action form along paths:

Proposition 2 Let $z$ be any point of $\mathbb{V}$ and $\gamma(\bar{z}, z)$ an arbitrary continuous path in $\mathbb{V}$ joining $\bar{z}$ to $z$. The line integral

$$
I(z)=\int_{\gamma(\bar{z}, z)} p \mathrm{~d} x
$$

only depends on the homotopy class $\check{z}$ of $\gamma(\bar{z}, z)$ and defines a phase of $\mathbb{V}$.

Proof. Let $\gamma^{\prime}(\bar{z}, z)$ be another path joining $\bar{z}$ to $z$ in $\mathbb{V}$ and homotopic to $\gamma(\bar{z}, z)$; the loop $\delta=\gamma(\bar{z}, z)-\gamma^{\prime}(\bar{z}, z)$ is thus homotopic to a point in $\mathbb{V}$. Let $h=h(s, t), 0 \leq s, t \leq 1$ be such a homotopy: $h(0, t)=\delta(t), h(1, t)=0$. As $s$ varies from 0 to 1 the loop $\delta$ will sweep out a two-dimensional surface $\mathcal{D}$ with boundary $\delta$ contained in $\mathbb{V}$. In view of the multi-dimensional Stokes theorem we have

$$
\int_{\delta} p \mathrm{~d} x=\iint_{\mathcal{D}} \mathrm{d} p \wedge \mathrm{d} x=0
$$


where the last equality follows from the fact that $\mathcal{D}$ is a subset of a Lagrangian manifold. It follows from this equality that

$$
\int_{\gamma(\bar{z}, z)} p \mathrm{~d} x=\int_{\gamma^{\prime}(\bar{z}, z)} p \mathrm{~d} x
$$

hence the integral of $p \mathrm{~d} x$ along $\gamma(\bar{z}, z)$ only depends on the homotopy class in $\mathbb{V}$ of the path joining $\bar{z}$ to $z$; it is thus a function of $\check{z} \in \check{\mathbb{V}}$. There remains to show that the function $\varphi: \check{\mathbb{V}} \longrightarrow \mathbb{R}$ defined by

$$
\varphi(\check{z})=\int_{\gamma(\bar{z}, z)} p \mathrm{~d} x
$$

is such that $\mathrm{d} \varphi(\check{z})=p \mathrm{~d} x$. The property being local, we can assume that $\mathbb{V}$ is simply connected, so that $\check{\mathbb{V}}=\mathbb{V}$. Since $\mathbb{V}$ is diffeomorphic to $\ell(z)=T_{z} \mathbb{V}$ in a neighborhood of $z$ we can reduce the proof to the case where $\mathbb{V}$ is a Lagrangian plane $\ell$. Let $A x+B p=0\left(A^{T} B=B A^{T}\right)$ be an equation of $\ell$, and

$$
\gamma(z): t \longmapsto\left(-B^{T} u(t), A^{T} u(t)\right) \quad, \quad 0 \leq t \leq 1
$$

be a differentiable curve starting from 0 and ending at $z=\left(-B^{T} u(1), A^{T} u(1)\right)$. We have

$$
\begin{aligned}
\varphi(z) & =\int_{\gamma(z)} p \mathrm{~d} x \\
& =-\int_{0}^{1} A^{T} u(t) \cdot B^{T} \dot{u}(t) \mathrm{d} t \\
& =-\int_{0}^{1} B A^{T} u(t) \cdot \dot{u}(t) \mathrm{d} t
\end{aligned}
$$

and hence, since $B A^{T}$ is symmetric:

$$
\varphi(z)=-\frac{1}{2} B A^{T} u(1)^{2}
$$

that is

$$
\mathrm{d} \varphi(z)=-B A^{T} u(1) \mathrm{d} u(1)=p \mathrm{~d} x .
$$

As already observed above we are slightly abusing language by calling $\varphi$ a "phase of $\mathbb{V}$ " since $\varphi$ is multi-valued on $\mathbb{V}$. This multi-valuedness is made explicit by studying the action of $\pi_{1}(\mathbb{V})$ on $\check{\mathbb{V}}$. The latter is defined as follows: let $\gamma$ be a loop in $\mathbb{V}$ with origin $z_{0}$ and $\check{\gamma} \in \pi_{1}(\mathbb{V})$ its homotopy 
class. Then $\check{\gamma} \check{z}$ is the homotopy class of the loop $\gamma$ followed by the path $\gamma(z)$ representing $\check{z}$. From the definition of the phase $\varphi$ follows that

$$
\varphi(\check{\gamma} \check{z})=\varphi(\check{z})+\oint_{\gamma} p \mathrm{~d} x .
$$

The phase is thus defined on $\mathbb{V}$ itself if and only if $\int_{\gamma} p \mathrm{~d} x=0$ for all loops in $\mathbb{V}$; this is the case if $\mathbb{V}$ is contractible. However Gromov has proved in [9] that if $\mathbb{V}$ is closed (i.e. compact and without boundary) then we cannot have $\oint_{\gamma} p \mathrm{~d} x=0$ for all loops $\gamma$ in $\mathbb{V}$; to construct the phase of such a manifold we thus have to use the procedure above.

\section{The Local Expression of the Phase}

Recall that a Lagrangian manifold which can be represented by an equation $p=\nabla_{x} \Phi(x)$ is called an "exact Lagrangian manifold". It turns out that Lagrangian manifolds are (locally) exact outside their caustic set, and this is most easily described in terms of the phase defined above. We use the following standard terminology: a point $z$ of a Lagrangian manifold $\mathbb{V}$ is called a "caustic point" if $z$ has no neighbourhood in $\mathbb{V}$ for which the restriction of the mapping $z=(x, p) \longmapsto x$ is a diffeomorphism; at a caustic point the tangent space $\ell(z)=T_{x} \mathbb{V}$ is the momentum space $0 \times \mathbb{R}_{p}^{n}$. The set $\Sigma$ of all caustic points of $\mathbb{V}$ is called the caustic of $\mathbb{V}$. Of course, caustics have no intrinsic meaning, whatsoever: there are just artefacts coming from the choice of a privileged $n$-dimensional plane (e.g., the configuration space) on which one projects the motion.

Let $\mathbb{U}$ be an open subset of $\mathbb{V}$ which contains no caustic points: $\mathbb{U} \cap \Sigma=$ $\emptyset$. Then the restriction $\chi_{\mathbb{U}}$ to $\mathbb{U}$ of the projection $\chi:(x, p) \longmapsto x$ is a diffeomorphism of $\mathbb{U}$ onto its image $\chi_{\mathbb{U}}(\mathbb{U})$, and $\left(\mathbb{U}, \chi_{\mathbb{U}}\right)$ is thus a local chart of $\mathbb{V}$. Choosing $\mathbb{U}$ small enough, we can assume that the fibre $\pi^{-1}(\mathbb{U})$ is the disjoint union of a family of open sets $\check{\mathbb{U}}$ in the universal covering of $\mathbb{V}$ and such that the restriction $\pi_{\mathbb{U}}$ to $\check{\mathbb{U}}$ of the projection $\pi: \check{\mathbb{V}} \longrightarrow \mathbb{V}$ is a diffeomorphism onto $\mathbb{U}$. It follows that $\left(\check{\mathbb{U}}, \chi_{\mathbb{U}} \circ \pi_{\mathbb{U}}\right)$ is a local chart of $\check{\mathbb{V}}$.

Proposition 3 Let $\Phi$ be the local expression of the phase $\varphi$ in any of the local charts $\left(\check{\mathbb{U}}, \chi_{\mathbb{U}} \circ \pi_{\mathbb{U}}\right)$ :

$$
\Phi(x)=\varphi\left(\left(\chi_{\mathbb{U}} \circ \pi_{\mathbb{U}}\right)^{-1}(x)\right) .
$$

The Lagrangian submanifold $\mathbb{U}$ is exact and can be represented by the equation

$$
p=\nabla_{x} \Phi(x)=\nabla_{x} \varphi\left(\left(\chi_{\mathbb{U}} \circ \pi_{\mathbb{U}}\right)^{-1}(x)\right) .
$$


Proof. Let us first show that the equation (9) remains unchanged if we

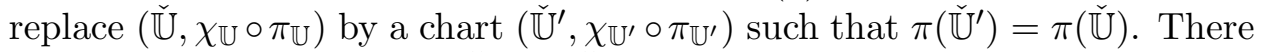
exists $\gamma \in \pi_{1}(\mathbb{V})$ such that $\check{U}^{\prime}=\gamma \check{\mathbb{U}}$ hence, by (7), the restrictions $\varphi_{\check{U}^{\prime}}$ and $\varphi_{\check{U}}$ differ by the constant

$$
C(\gamma)=\oint_{\gamma} p \mathrm{~d} x
$$

It follows that

$$
\nabla_{x} \varphi\left(\left(\chi_{\mathbb{U}^{\prime}} \circ \pi_{\mathbb{U}^{\prime}}\right)^{-1}(x)\right)=\nabla_{x} \varphi\left(\left(\chi_{\mathbb{U}} \circ \pi_{\mathbb{U}}\right)^{-1}(x)\right)
$$

and hence the right-hand side of the identity (9) does not depend on the choice of local chart $\left(\check{\mathbb{U}}, \chi_{\mathbb{U}} \circ \pi_{\mathbb{U}}\right)$. Set now $\left(\chi_{\mathbb{U}} \circ \pi_{\mathbb{U}}\right)^{-1}(x)=(p(x), x)$; we have, for $x \in \chi_{\mathbb{U}} \circ \pi_{\mathbb{U}}(\mathbb{U})$,

$$
\mathrm{d} \Phi(x)=\mathrm{d} \varphi(p(x), x)=p(x) \mathrm{d} x
$$

hence (9).

\section{Symplectic Frames and Lagrangian Phases}

The observant reader will have noticed that the phase of a Lagrangian manifold was defined in terms of one special coordinate system, namely the canonical coordinates $x, p$. It is of course of interest to determine what happens to the phase under symplectic changes of variables. Let us introduce, following Leray [12, the notion of symplectic frame: by definition, a symplectic frame is any pair $\left(\ell, \ell^{*}\right)$ of Lagrangian planes such that $\mathbb{R}_{z}^{2 n}=\ell \oplus \ell^{*}$; equivalently: $\ell \cap \ell^{*}=0$. Set $\ell_{x}=\mathbb{R}_{x}^{n} \times 0$ and $\ell_{p}=0 \times \mathbb{R}_{p}^{n}$ (the configuration space, and the momentum space, respectively). The pair $\left(\ell_{x}, \ell_{p}\right)$ is a symplectic frame: we call it the canonical frame. The symplectic group acts transitively on all pairs of transverse Lagrangian planes (see [5, 7]); it follows that the image $S\left(\ell, \ell^{*}\right)=\left(S \ell, S \ell^{*}\right)$ of a symplectic frame is a symplectic frame, and that for every pair $\left(\ell, \ell^{*}\right),\left(\ell^{\prime}, \ell^{\prime *}\right)$ of symplectic frame there exists $R \in \operatorname{Sp}(n)$ such that $\left(\ell, \ell^{*}\right)=R\left(\ell^{\prime}, \ell^{\prime *}\right)$ (i.e. $\ell=R \ell^{\prime}$ and $\left.\ell^{*}=R \ell^{\prime *}\right)$. We will call such an $R$ a symplectic change of frame; a manifold which is Lagrangian in one such frame is Lagrangian in all symplectic frames and we will see that there is an intrinsic (i.e. frame-independent) function on $\check{\mathbb{V}}$ which we call, again following Leray, the Lagrangian phase of $\mathbb{V}$.

For the sake of notational brevity we will omit the dot · for scalar products and write, for instance, $p x$ in place of $p \cdot x$.

Let $\operatorname{Sp}(n)$ be the symplectic group: $S \in \operatorname{Sp}(n)$ if and only if $S$ is a linear automorphism of $\mathbb{R}_{z}^{2 n}$ preserving the symplectic form $\sigma: \sigma\left(S z, S z^{\prime}\right)=$ 
$\sigma\left(z, z^{\prime}\right)$ for all vectors $z, z^{\prime}$. For every $S \in \operatorname{Sp}(n)$ the image $S(\mathbb{V})$ is also a Lagrangian manifold. The following result allows us to compare the phases of $\mathbb{V}$ and $S(\mathbb{V})$; it will also allow us to give a frame-independent definition of the phase of $\mathbb{V}$.

Proposition 4 For $S \in \operatorname{Sp}(n)$ set $\left(x_{S}, p_{S}\right)=S(x, p)$. (*) We have

$$
p_{S} \mathrm{~d} x_{S}-x_{S} \mathrm{~d} p_{S}=p \mathrm{~d} x-x \mathrm{~d} p .
$$

(**) Define a function $\varphi_{S}: \check{\mathbb{V}} \longrightarrow \mathbb{R}$ by the formula

$$
\varphi_{S}(\check{z})=\varphi(\check{z})+\frac{1}{2}\left(p_{S} x_{S}-p x\right) .
$$

That function is differentiable, and we have

$$
\mathrm{d} \varphi_{S}(\check{z})=p_{S} \mathrm{~d} x_{S} \quad \text { if } \pi(\check{z})=(x, p) .
$$

Proof. (*) Writing $S$ in block-matrix form

$$
S=\left(\begin{array}{ll}
A & B \\
C & D
\end{array}\right)
$$

the condition that $S$ is symplectic implies that $A^{T} C$ and $B^{T} D$ are symmetric, and that $A^{T} D-C^{T} B=I$. Setting $x_{S}=A x+B p, p_{S}=C x+D p$, and expanding the products, we get

$$
\begin{aligned}
p_{S} \mathrm{~d} x_{S}-x_{S} \mathrm{~d} p_{S} & =\left(A^{T} C x+A^{T} D p-C^{T} A x-C^{T} B p\right) \mathrm{d} x+ \\
& \left(B^{T} C x+B^{T} D p-D^{T} A x-D^{T} B p\right) \mathrm{d} p \\
& =p \mathrm{~d} x-x \mathrm{~d} p
\end{aligned}
$$

proving (101). (Notice that in general we do not have $p_{S} \mathrm{~d} x_{S}=p \mathrm{~d} x$.). (**) Differentiating the right-hand side of (11) we get, since $\mathrm{d} \varphi(\check{z})=p \mathrm{~d} x$,

$$
\begin{aligned}
\mathrm{d} \varphi_{S}(\check{z}) & =\frac{1}{2}(p \mathrm{~d} x-x \mathrm{~d} p)+\frac{1}{2} \mathrm{~d}\left(p_{S} x_{S}\right) \\
& =\frac{1}{2}\left(p_{S} \mathrm{~d} x_{S}-x_{S} \mathrm{~d} p_{S}\right)+\frac{1}{2} \mathrm{~d}\left(p_{S} x_{S}\right) \\
& =p_{S} \mathrm{~d} x_{S}
\end{aligned}
$$

which proves (12).

We can identify the universal covering of $S(\mathbb{V})$ with that, $\check{\mathbb{V}}$, of $\mathbb{V}$ : for this it suffices to define the projection

$$
\pi_{S}=S \circ \pi: \check{\mathbb{V}} \longrightarrow S(\mathbb{V}): \pi_{S}(\check{z})=S z=\left(x_{S}, p_{S}\right) .
$$

Proposition 4 can then be restated as follows: 
The phase of $S(V)$ is the function $\varphi_{S}: \check{V} \longrightarrow R$ defined by formula (11): we have $d \varphi_{S}(\check{z})=p d x$ if $\pi_{S}(\check{z})=(x, p)$.

We will call "Lagrangian phase of $\mathbb{V}$ " the function $\lambda: \check{\mathbb{V}} \longrightarrow \mathbb{R}$ defined by

$$
\lambda(\check{z})=\varphi(\check{z})-\frac{1}{2} p x \text { if } \pi(\check{z})=(x, p) .
$$

In view of Lemma 4 the invariant phase $\lambda_{R}$ of the Lagrangian manifold $R \mathbb{V}$ is

$$
\lambda_{R}(\check{z})=\varphi_{R}(\check{z})-\frac{1}{2} p_{R} x_{R} \text { if } \pi_{R}(\check{z})=(x, p) ;
$$

since in view of formula (11) we have

$$
\varphi_{R}(\check{z})=\varphi(\check{z})+\frac{1}{2}\left(p_{R} x_{R}-p x\right)
$$

it follows that $\lambda_{R}(\check{z})=\lambda(\check{z})$ : the Lagrangian phase is thus the same in all symplectic frames.

Notice that it follows from definition (13) that the differential of the Lagrangian phase is

$$
\mathrm{d} \lambda(\check{z})=\frac{1}{2}(p \mathrm{~d} x-x \mathrm{~d} p) .
$$

Let us note the following particular case of Lemma 4 assume that $S$ is a free symplectic matrix, that is

$$
S=\left(\begin{array}{ll}
A & B \\
C & D
\end{array}\right) \quad, \quad \operatorname{det} B \neq 0
$$

(equivalently, $\left.S\left(0 \times \mathbb{R}_{p}^{n}\right) \cap\left(0 \times \mathbb{R}_{p}^{n}\right)=0\right)$. Then $S$ admits a homogeneous free generating function

$$
W\left(x, x^{\prime}\right)=\frac{1}{2} B^{-1} A x^{2}-B^{-1} x x^{\prime}+\frac{1}{2} D B^{-1} x^{\prime 2}
$$

(where $B^{-1} A x^{2}=B^{-1} A x \cdot x$, etc.), and we have $\left(x_{S}, p_{S}\right)=S(x, p)$ if and only if $p_{S}=\nabla_{x} W\left(x_{S}, x\right)$ and $p=-\nabla_{x} W\left(x_{S}, x\right)$. Since $W$ is homogeneous of degree two in the $x, x^{\prime}$ variables, Euler's formula yields

$$
\begin{aligned}
W\left(x_{S}, x\right) & =\frac{1}{2}\left(x_{S} \nabla_{x_{S}} W\left(x_{S}, x\right)+x \nabla_{x} W\left(x_{S}, x\right)\right) \\
& =\frac{1}{2}\left(p_{S} x_{S}-p x\right)
\end{aligned}
$$

hence formula (11) can be rewritten as

$$
\varphi_{S}(\check{z})=\varphi(\check{z})+W\left(x_{S}, x\right) .
$$

As we will see in Section [6 formula (16) is a particular case of a more general result describing the action of Hamiltonian flows on the phase of a Lagrangian manifold. 


\section{Hamiltonian Motions and Phase}

We are now going to investigate the action of Hamiltonian flows on the phase. Let us first introduce some notations. Let $H=H(z, t)$ ("the Hamiltonian") be a smooth real function defined on $\mathbb{R}_{z}^{2 n} \times \mathbb{R}_{t}$. We denote by $\left(f_{t, t^{\prime}}^{H}\right)$ the time-dependent flow it determines: for an initial point $z^{\prime}$ set $z_{t}=f_{t, t^{\prime}}^{H}\left(z_{t^{\prime}}\right)$; the function $t \longmapsto z_{t}$ is the solution of Hamilton's equations

$$
\dot{x}=\nabla_{p} H(z, t) \quad, \quad \dot{p}=-\nabla_{x} H(z, t)(z, t)
$$

passing through $z_{t^{\prime}}^{\prime}$ at time $t^{\prime}$. Notice that $f_{t, t^{\prime}}^{H} \circ f_{t^{\prime}, t^{\prime \prime}}^{H}=f_{t, t^{\prime \prime}}^{H}$. The suspended Hamiltonian flow $\left(\tilde{f}_{t}^{H}\right)$ is defined on the extended phase space $\mathbb{R}_{z}^{2 n} \times \mathbb{R}_{t}$; it is defined by

$$
\tilde{f}_{t}^{H}\left(z^{\prime}, t^{\prime}\right)=\left(f_{t+t^{\prime}, t^{\prime}}^{H}\left(z^{\prime}\right), t+t^{\prime}\right)
$$

and is the flow of the suspended Hamiltonian vector field

$$
\tilde{X}_{H}=\left(\nabla_{p} H,-\nabla_{x} H, 1\right)
$$

we will also use the notation

$$
f_{t}^{H}=f_{t, 0}^{H}
$$

and call, somewhat sloppily, the family of canonical transformations $\left(f_{t}^{H}\right)$ the flow determined by $H$ (it is not truly a flow when $H$ is effectively timedependent: $f_{t}^{H} f_{t^{\prime}}^{H} \neq f_{t+t^{\prime}}^{H}$.

We will use the properties of the Poincaré-Cartan integral form. It is the one-form $\alpha_{H}$ on $\mathbb{R}_{z}^{2 n} \times \mathbb{R}_{t}$ defined by

$$
\alpha_{H}=p \mathrm{~d} x-H \mathrm{~d} t .
$$

Its interest comes from the following property which expresses the fact $\alpha_{H}$ is a relative integral invariant (see [1, 13]): the contraction of the exterior derivative

$$
\mathrm{d} \alpha_{H}=\mathrm{d} p \wedge \mathrm{d} x-\mathrm{d} H \wedge \mathrm{d} t
$$

with the suspended Hamilton vector field $\tilde{X}_{H}$ is zero: $i_{\tilde{X}_{H}} \mathrm{~d} \alpha_{H}=0$. This means that for every vector $\tilde{Y}(z, t)$ in $\mathbb{R}_{z}^{2 n} \times \mathbb{R}_{t}$ originating at a point $(z, t)$ we will have

$$
\mathrm{d} \alpha_{H}\left(\tilde{X}_{H}(z, t), \tilde{Y}(z, t)\right)=0 .
$$

This property has the following, for us very important, consequence: let $\tilde{\gamma}:[0,1] \longrightarrow \mathbb{R}_{z}^{2 n} \times \mathbb{R}_{t}$ be a smooth curve in extended phase space on which 
we let the suspended flow $\tilde{f}_{t}^{H}$ act; as time varies, $\tilde{\gamma}$ will sweep out a twodimensional surface $\Sigma_{t}$ whose boundary $\partial \Sigma_{t}$ consists of $\tilde{\gamma}, \tilde{f}_{t}^{H}(\tilde{\gamma})$, and two arcs of phase-space trajectory, $\tilde{\gamma}_{0}$ and $\tilde{\gamma}_{1}: \tilde{\gamma}_{0}$ is the trajectory of the origin $\tilde{\gamma}(0)$ of $\tilde{\gamma}$, and $\tilde{\gamma}_{1}$ that of its endpoint $\tilde{\gamma}(1)$. It turns out that we will have

$$
\int_{\partial \Sigma_{t}} \alpha_{H}=0
$$

Here is a sketch of the proof: using the multi-dimensional Stokes formula we have

$$
\int_{\partial \Sigma_{t}} \alpha_{H}=\int_{\Sigma_{t}} \mathrm{~d} \alpha_{H}
$$

Since the surface $\Sigma_{t}$ consists of flow lines of $\tilde{X}_{H}$ each pair $(\tilde{X}, \tilde{Y})$ of tangent vectors at a point $(z, t)$ can be written as a linear combination of two independent vectors, and one of these vectors can be chosen as $\tilde{X}_{H}$. It follows that $\mathrm{d} \alpha_{H}(\tilde{X}, \tilde{Y})$ is a sum of terms of the type $\mathrm{d} \alpha_{H}\left(\tilde{X}_{H}, \tilde{Y}\right)$, which are equal to zero in view of (17). We thus have $\int_{\Sigma_{t}} \mathrm{~d} \alpha_{H}=0$, whence (18).

From now on we will use the definite integral notation

$$
\int_{z_{t^{\prime}}}^{z_{t}} \alpha_{H}=\int_{z_{t^{\prime}}}^{z_{t}} p \mathrm{~d} x-H \mathrm{~d} t
$$

for the integral of the Poincaré-Cartan form along the phase space trajectory $s \longmapsto f_{s, t^{\prime}}^{H}\left(z_{t^{\prime}}\right)$ joining $z_{t^{\prime}}$ to $z_{t}=f_{t, t^{\prime}}^{H}\left(z_{t^{\prime}}\right)$. Let $\mathbb{V}$ be a Lagrangian manifold and $\mathbb{V}_{t}=f_{t}^{H}(\mathbb{V})$. Notice that $\mathbb{V}_{t}=f_{t, t^{\prime}}^{H}\left(\mathbb{V}_{t^{\prime}}\right)$. Since Hamiltonian flows consist of symplectomorphisms each $\mathbb{V}_{t}$ is a Lagrangian manifold, and the function $\varphi_{t}: \check{\mathbb{V}}_{t} \longrightarrow \mathbb{R}$ defined by

$$
\varphi_{t}\left(\check{z}_{t}\right)=\int_{\gamma\left(\bar{z}_{t}, z_{t}\right)} p \mathrm{~d} x
$$

$\left(\check{z}_{t}\right.$ being the homotopy class in $\mathbb{V}_{t}$ of a path $\gamma\left(\bar{z}_{t}, z_{t}\right)$ ) obviously is a phase when $\bar{z}_{t}=f_{t}^{H}\left(\bar{z}_{0}\right)$ is chosen as base point in $\mathbb{V}_{t}$. The following result relates $\varphi_{t}$ to the phase $\varphi=\varphi_{0}$ of $\mathbb{V}=\mathbb{V}_{0}$ :

Lemma 5 Let $\check{z}=\check{z}_{0}$ be a point in $\mathbb{V}$ and $\check{z}_{t}$ its image in $\mathbb{V}_{t}$ (i.e. $\check{z}_{t}$ is the homotopy class in $\mathbb{V}_{t}$ of the image by $f_{t}^{H}$ of a path representing $\left.\check{z}\right)$. We have

$$
\varphi_{t}\left(\check{z}_{t}\right)-\varphi(\check{z})=\int_{\bar{z}_{0}}^{\bar{z}_{t}} \alpha_{H}-\int_{z_{0}}^{z_{t}} \alpha_{H} .
$$


Proof. Let $\Sigma_{t}$ be the closed curve

$$
\Sigma_{t}=\left[\bar{z}_{0}, \bar{z}_{t}\right]+\gamma\left(\bar{z}_{t}, z_{t}\right)-\left[z_{0}, z_{t}\right]-\gamma\left(\bar{z}_{0}, z_{0}\right)
$$

where $\left[\bar{z}_{0}, \bar{z}_{t}\right]$ (resp. $\left[z_{0}, z_{t}\right]$ ) is the Hamiltonian trajectory joining $\bar{z}_{0}$ to $\bar{z}_{t}$ (resp. $z_{0}$ to $z_{t}$ ). In view of the consequence (18) of the relative invariance property of the Poincaré-Cartan form $\alpha_{H}$ we have

$$
\int_{\Sigma_{t}} \alpha_{H}=0
$$

Since $\mathrm{d} t=0$ along both $\gamma\left(\bar{z}_{t}, z_{t}\right)$ and $\gamma\left(\bar{z}_{0}, z_{0}\right)$ we have

$$
\int_{\gamma\left(\bar{z}_{t}, z_{t}\right)} \alpha_{H}=\int_{\gamma\left(\bar{z}_{t}, z_{t}\right)} p \mathrm{~d} x \quad, \quad \int_{\gamma\left(\bar{z}_{0}, z_{0}\right)} \alpha_{H}=\int_{\gamma\left(\bar{z}_{0}, z_{0}\right)} p \mathrm{~d} x
$$

and hence (20) is equivalent to

$$
\int_{\gamma\left(\bar{z}_{0}, z_{0}\right)} p \mathrm{~d} x+\int_{z_{0}}^{z_{t}} \alpha_{H}-\int_{\gamma\left(\bar{z}_{t}, z_{t}\right)} p \mathrm{~d} x+\int_{\bar{z}_{0}}^{\bar{z}_{t}} \alpha_{H}=0
$$

that is to (19).

Lemma [5 has the following fundamental consequence for the phase of $\mathbb{V}_{t}=f_{t}^{H}(\mathbb{V})$ :

Proposition 6 Set $z_{0}=z$ and $\check{z}=\check{z}_{0}$. The function $\varphi(\cdot, t): \check{\mathbb{V}} \longrightarrow \mathbb{R}$ defined by

$$
\varphi(\check{z}, t)=\varphi(\check{z})+\int_{z, 0}^{z_{t}, t} \alpha_{H} \quad, \quad z_{t}=f_{t}^{H}(z)
$$

is a phase of $\mathbb{V}_{t}$ : for fixed $t$ we have

$$
\mathrm{d} \varphi(\check{z}, t)=p_{t} \mathrm{~d} x_{t} \quad \text { if } \pi_{t}(\check{z})=z_{t}=\left(x_{t}, p_{t}\right)
$$

that is, equivalently,

$$
\mathrm{d} \varphi(\check{z}, t)=p_{t} \mathrm{~d} x_{t} \quad \text { if } \pi(\check{z})=z=(x, p) .
$$

Proof. In view of Lemma 5 the function $\varphi(\cdot, t)$ satisfies

$$
\varphi(\check{z}, t)=\varphi_{t}\left(\check{z}_{t}\right)+\int_{\bar{z}_{0}}^{\bar{z}_{t}} \alpha_{H}
$$

where $\check{z}_{t} \in \check{\mathbb{V}}_{t}$ is the image of $\check{z}$ by $f_{t}^{H}$. It follows that for fixed $t$ we have

$$
\mathrm{d} \varphi(\check{z}, t)=p_{t} \mathrm{~d} x_{t} \text { if } \pi_{t}\left(\check{z}_{t}\right)=\left(x_{t}, p_{t}\right)
$$


where $\pi_{t}: \check{\mathbb{V}}_{t} \longrightarrow \mathbb{V}_{t}$ is the projection $\check{z}_{t} \longmapsto z_{t}$.

We will call the function $\varphi(\cdot, t): \check{\mathbb{V}} \longrightarrow \mathbb{R}$ the phase of $\mathbb{V}_{t}$; observe that it is defined, not on $\check{\mathbb{V}}_{t}$, but on $\check{\mathbb{V}}$ itself, viewed as a "master universal covering manifold".

The following particular case relates the Hamiltonian phase to Proposition 4 on changes of symplectic frames:

Corollary 7 Let $H$ be a Hamiltonian which is quadratic and homogeneous in the position and momentum variables; its flow thus consists of symplectic matrices $S_{t}^{H}$. The Hamiltonian phase of $S_{t}^{H}(\mathbb{V})$ is

$$
\varphi(\check{z}, t)=\varphi(\check{z})+\frac{1}{2}\left(p_{t} x_{t}-p x\right) .
$$

Proof. Since $H$ is quadratic we have, using successively Euler's formula and Hamilton's equations,

$$
\begin{aligned}
H\left(z_{t}, t\right) & =\frac{1}{2}\left(x_{t} \nabla_{x} H\left(z_{t}, t\right)+p_{t} \nabla_{p} H\left(z_{t}, t\right)\right) \\
& =\frac{1}{2}\left(-x_{t} \dot{p}_{t}+p_{t} \dot{x}_{t}\right)
\end{aligned}
$$

and hence

$$
\begin{aligned}
\int_{z_{0}}^{z_{t}} \alpha_{H} & =\frac{1}{2} \int_{0}^{t}\left(p_{s} \dot{x}_{s}+x_{s} \dot{p}_{s}\right) \mathrm{d} s \\
& =\frac{1}{2}\left(p_{t} x_{t}-p x\right)
\end{aligned}
$$

whence (25) in view of (21).

Another interesting particular case of Proposition [6] occurs when the Lagrangian manifold $\mathbb{V}$ is invariant under the flow: $f_{t}^{H}(\mathbb{V})=\mathbb{V}$. (This situation typically occurs when one has a completely integrable system and $\mathbb{V}$ is an associated Lagrangian torus.)

Corollary 8 Let $H$ be a time-independent Hamiltonian, $\left(f_{t}^{H}\right)$ its flow, and assume that $\mathbb{V}$ is invariant under $\left(f_{t}^{H}\right)$ (that is $f_{t}^{H}(\mathbb{V})=\mathbb{V}$ for all $t$ ). If $\check{z}$ is the homotopy class in $\mathbb{V}$ of a a path $\gamma\left(\bar{z}_{0}, z\right)$ and $\gamma\left(z, z_{t}\right)$ is the piece of Hamiltonian trajectory joining $z$ to $z_{t}$ then

$$
\varphi(\check{z}, t)=\varphi\left(\check{z}_{t}\right)-E t
$$

where $E$ is the (constant) value of $H$ on $\mathbb{V}$ and $\check{z}_{t}$ the homotopy class of the path $\gamma\left(\bar{z}_{0}, z\right)+\gamma\left(z, z_{t}\right)$ in $\mathbb{V}$. 
Proof. The trajectory $s \longmapsto z_{s}=f_{s}^{H}(z)$ is a path $\gamma\left(z, z_{t}\right)$ in $\mathbb{V}$ joining $z$ to $z_{t}$ hence

$$
\int_{\gamma\left(\bar{z}_{0}, z\right)} p \mathrm{~d} x+\int_{z}^{z_{t}} \alpha_{H}=\int_{\gamma\left(\bar{z}_{0}, z_{t}\right)} p \mathrm{~d} x-E t
$$

where $\gamma\left(\bar{z}_{0}, z_{t}\right)=\gamma\left(\bar{z}_{0}, z\right)+\gamma\left(z, z_{t}\right)$. The result follows since the first integral in the right-hand side of this equality is by definition $\varphi\left(\check{z}_{t}\right)$.

Proposition 6 allows us also to link the notion of phase of a Lagrangian manifold to the standard Hamilton-Jacobi theory.

Proposition 9 Let $z \in \mathbb{V}$ have a neighborhood $\mathbb{U}$ in $\mathbb{V}$ projecting diffeomorphically on $\mathbb{R}_{x}^{n}$. (*) There exists $\varepsilon>0$ such that the local expression $\Phi=\Phi(x, t)$ of the phase $\varphi$ is defined for $|t|<\varepsilon$ and (**) $\Phi$ satisfies the Hamilton-Jacobi equation

$$
\frac{\partial \Phi}{\partial t}+H\left(x, \nabla_{x} \Phi\right)=0
$$

for $|t|<\varepsilon$.

Proof. The first part $(*)$ is an immediate consequence of Proposition (3) (the existence of $\varepsilon$ follows from the fact that the caustic is a closed subset of $\mathbb{V})$. To prove $(* *)$ we observe that

$$
\Phi(x, t)=\Phi\left(x^{\prime}, 0\right)+\int_{z^{\prime}, 0}^{z, t} p \mathrm{~d} x-H \mathrm{~d} t
$$

in view of formula (21) in Proposition [6] now we can parametrize the arc joining $z^{\prime}, 0$ to $z, t$ by $x$ and $t$ hence

$$
\Phi(x, t)=\Phi\left(x^{\prime}, 0\right)+\int_{x^{\prime}, 0}^{x, t} p \mathrm{~d} x-H \mathrm{~d} t
$$

which is precisely the solution of Hamilton-Jacobi's equation with initial datum $\Phi$ at time $t=0$ (cf. formula (3) ).

\section{Phase and Heisenberg-Weyl Operators}

Let $T\left(z_{a}\right)$ be the phase space translation $z \longmapsto z+z_{a}$. That operator can be viewed as the time-one map of the flow determined by the "translation Hamiltonian $H^{a}=\sigma\left(z, z_{a}\right)$ : that flow consists of the mappings $f_{t}^{a}(z)=$ $z+t z_{a}$, and thus $T\left(z_{a}\right)=f_{1}^{a}$. In Proposition [10 below this is taken into account, that is, the phases of the translated Lagrangian manifolds will be calculated using formula (21) of Proposition [6] above. 
Proposition 10 Let $T\left(z_{a}\right)$ be the translation with vector $z_{a}=\left(x_{a}, p_{a}\right)$ and $T\left(z_{b}\right)$ that with vector $z_{b}=\left(x_{b}, p_{b}\right)$. (*) The phase $\varphi_{a}$ of $T\left(z_{a}\right) \mathbb{V}$ is given by

$$
\varphi_{a}(\check{z})=\varphi(\check{z})+\frac{1}{2} p_{a} x_{a}+p_{a} x_{0} \quad \text { if } \pi(\check{z})=(x, p) .
$$

$(* *)$ Let $\varphi_{a, b}$ be the phase of $T\left(z_{a}\right)\left(T\left(z_{b}\right) \mathbb{V}\right)$ and $\varphi_{a+b}$ that of $T\left(z_{a}+z_{b}\right) \mathbb{V}$; we have

$$
\varphi_{a, b}(\check{z})-\varphi_{a+b}(\check{z})=-\frac{1}{2} \sigma\left(z_{a}, z_{b}\right)
$$

and hence

$$
\varphi_{a, b}(\check{z})-\varphi_{b, a}(\check{z})=\sigma\left(z_{a}, z_{b}\right) .
$$

Proof. We have, in view of (24),

$$
\begin{aligned}
\varphi_{a}(\check{z}) & =\varphi\left(\check{z}_{0}\right)+\int_{0}^{1}\left(p_{0}+t p_{a}\right) x_{a} \mathrm{~d} t-\int_{0}^{1} \sigma\left(z_{0}+t z_{a}, z_{a}\right) \mathrm{d} t \\
& =\varphi\left(\check{z}_{0}\right)+p_{0} x_{a}+\frac{1}{2} p_{a} x_{a}-\left(p_{0} x_{a}-p_{a} x_{0}\right) \\
& =\varphi\left(\check{z}_{0}\right)+\frac{1}{2} p_{a} x_{a}+p_{a} x_{0}
\end{aligned}
$$

whence (27). Formulae (28) follows from (27) since we have

$$
\varphi_{a, b}(\check{z})=\left(\frac{1}{2} p_{b} x_{b}+p_{b} x_{0}\right)+\left(\frac{1}{2} p_{a} x_{a}+p_{a}\left(x_{b}+x_{0}\right)\right)
$$

and

$$
\varphi_{a+b}(\check{z})=\frac{1}{2}\left(p_{a}+p_{b}\right)\left(x_{a}+x_{b}\right)+\left(p_{a}+p_{b}\right) x_{0} .
$$

Formula (29) follows from formula (28).

Remark 11 The phases of $T\left(z_{a}\right)\left(T\left(z_{b}\right) \mathbb{V}\right)$ and $T\left(z_{a}+z_{b}\right) \mathbb{V}$ are different, even though these manifolds are the same! In fact, formula (28) shows that the difference between the phases of $T\left(z_{a}+z_{b}\right) \mathbb{V}$ and $T\left(z_{a}\right)\left(T\left(z_{b}\right) \mathbb{V}\right)$ is just (up to the sign) the area of the phase space triangle spanned by the vectors $z_{a}, z_{b}$ (see the discussion and Fig.3, p.211 in Littlejohn [14]).

We also have the following "symplectic covariance" result:

Proposition 12 The Hamiltonian phases of the identical Lagrangian manifolds $S_{t}^{H}\left(T\left(z_{a}\right) \mathbb{V}\right)$ and $T\left(S_{t}^{H}\left(z_{a}\right)\right) S_{t}^{H} \mathbb{V}$ are equal.

Proof. The phase of $T\left(z_{a}\right) \mathbb{V}$ is

$$
\varphi_{a}(\check{z})=\varphi\left(\check{z}_{0}\right)+\frac{1}{2} p_{a} x_{a}+p_{a} x_{0}
$$


hence that of $S_{t}^{H}\left(T\left(z_{a}\right) \mathbb{V}\right.$ ) is (using (25) and the linearity of $S_{t}^{H}$ ):

$$
\begin{aligned}
A(t)=\varphi\left(\check{z}_{0}\right)+\frac{1}{2} p_{a} x_{a}+ & p_{a} x_{0}+ \\
& \left.\frac{1}{2}\left(p_{0, t}+p_{a, t}\right)\left(x_{0, t}+x_{a, t}\right)-\frac{1}{2}\left(p_{0}+p_{a}\right)\left(x_{0}+x_{a}\right)\right)
\end{aligned}
$$

where $z_{0, t}=S_{t}^{H} z_{0}, z_{a, t}=S_{t}^{H} z_{a}$. Similarly, the Hamiltonian phase of $S_{t}^{H} \mathbb{V}$ is

$$
\varphi(\check{z}, t)=\varphi\left(\check{z}_{0}\right)+\frac{1}{2}\left(p_{0, t} x_{0, t}-p_{0} x_{0}\right)
$$

hence that of $T\left(S_{t}^{H}\left(z_{a}\right)\right) S_{t}^{H} \mathbb{V}$ is

$$
B(t)=\varphi\left(\check{z}_{0}\right)+\frac{1}{2}\left(p_{0, t} x_{0, t}-p_{0} x_{0}\right)+\frac{1}{2} p_{a, t} x_{a, t}+p_{a, t} x_{0, t}
$$

and thus

$$
\begin{aligned}
A(t)-B(t) & =\frac{1}{2}\left(p_{a} x_{0}-p_{0} x_{a}\right)-\frac{1}{2}\left(p_{a, t} x_{0, t}-p_{0, t} x_{a, t}\right) \\
& =\frac{1}{2}\left(\sigma\left(z_{a}, z_{0}\right)-\sigma\left(z_{a, t}, z_{0, t}\right)\right) \\
& =\frac{1}{2}\left(\sigma\left(z_{a}, z_{0}\right)-\sigma\left(S_{t}^{H} z_{a}, S_{t}^{H} z_{0}\right)\right) .
\end{aligned}
$$

Since $S_{t}^{H} \in \operatorname{Sp}(n)$ we have $\sigma\left(S_{t}^{H} z_{a}, S_{t}^{H} z_{0}\right)=\sigma\left(z_{a}, z_{0}\right)$ and hence $A(t)=$ $B(t)$, proving the Proposition.

Let us extend the results above to the case of a Hamiltonian flow "displacing" points in the direction of the field of tangents to a smooth curve in phase space. To such a curve $t \longmapsto \gamma(t)=\left(x^{\gamma}(t), p^{\gamma}(t)\right)$ we associate the time-dependent Hamiltonian $H^{\gamma}$ defined by

$$
H^{\gamma}(z, t)=\sigma(z, \dot{\gamma}(t))=p \dot{x}^{\gamma}(t)-x \dot{p}^{\gamma}(t) .
$$

The solutions of the associated Hamilton equations $\dot{x}(t)=\dot{x}^{\gamma}(t), \dot{p}(t)=\dot{p}^{\gamma}(t)$ are given by

$$
z_{t}=z(0)+\gamma(t)-\gamma(0)
$$

hence the flow $\left(f_{t, t^{\prime}}^{\gamma}\right)$ propagates points along curves which are translations of $\gamma$. We will therefore call $H^{\gamma}$ the displacement Hamiltonian along $\gamma$. Set $f_{t}^{\gamma}=f_{t, 0}^{\gamma}$ and let $\mathbb{V}$ be a Lagrangian manifold with phase $\varphi$.

Proposition 13 The phase of the displaced Lagrangian manifold $\mathbb{V}^{\gamma(t)}=$ $f_{t, 0}^{\gamma}(\mathbb{V})$ is

$$
\varphi_{\gamma}(\check{z}, t)=\varphi(\check{z})+\frac{1}{2}\left(p_{t} x_{t}-p_{0} x_{0}\right)-\frac{1}{2} \int_{\gamma} p \mathrm{~d} x-x \mathrm{~d} p .
$$

If, in particular, $\gamma$ is a loop then

$$
\varphi_{\gamma}(\check{z}, t)=\varphi(\check{z})-\frac{1}{2} \int_{\gamma} p \mathrm{~d} x-x \mathrm{~d} p .
$$


Proof. In view of formula (21) the phase of $\mathbb{V}^{\gamma(t)}$ is

$$
\varphi(\check{z}, t)=\varphi(\check{z})+\int_{0}^{t}(p(s) \dot{x}(s)-\sigma(z(s), \dot{\gamma}(s))) \mathrm{d} s ;
$$

we have $\sigma(z(s), \dot{\gamma}(s))=\sigma(z(s), \dot{z}(s))$ and hence

$$
p(s) \dot{x}(s)-\sigma(z(s), \dot{\gamma}(s))=\dot{p}(s) x(s) .
$$

Noting that

$$
\begin{aligned}
\dot{p}(s) x(s) & =\frac{1}{2}(p(s) \dot{x}(s)+\dot{p}(s) x(s)-(p(s) \dot{x}(s)-\dot{p}(s) x(s))) \\
& =\frac{1}{2} \frac{\mathrm{d}}{\mathrm{d} t}(p(s) x(s))-\frac{1}{2} \sigma(z(s), \dot{z}(s))
\end{aligned}
$$

and integrating we get formula (30).

The result above can actually be recovered from Proposition (10) by using infinitesimal translations: segmenting the trajectory $t \longmapsto z(t)$ into straight sections $\left[z, z_{1}\right],\left[z_{1}, z_{2}\right], \ldots$ where $z_{k}=z(k \Delta t)(\Delta t=t / N)$, one finds that the limit of the product of these operators is precisely

$$
\lim _{N \rightarrow \infty} T\left(z_{N}-z_{N-1}\right) \cdots T\left(z_{2}-z_{1}\right) T\left(z_{1}-z\right)=T^{\gamma(t)} .
$$

(This observation thus a posteriori justifies formula (3.27), p.212, in Littlejohn [14.)

Both formulas (28), (29) in Proposition[10 are strongly reminiscent of the commutation formulas in the quantum-mechanical Heisenberg-Weyl group; however there is nothing quantum mechanical involved in our constructions! Let us discuss this point in some detail. Recall (see for instance Littlejohn [14] ) that the basic idea of the Heisenberg-Weyl operators is that they move wave functions around in phase space. This is done as follows: for a given quantum state $|\Psi\rangle$ the position and momentum expectation values are $\langle x\rangle$ and $\langle p\rangle$; this can be written collectively as $\langle z\rangle=\langle\Psi|z| \Psi\rangle$. Heisenberg-Weyl operators $\hat{T}\left(z_{a}\right)$ are parameterized by points $z_{a}$ in phase space, and have the property that if $|\Psi\rangle$ has the expectation value $\langle z\rangle$ then $\hat{T}\left(z_{a}\right)|\Psi\rangle$ should have the expectation value $\langle z\rangle+z_{a}$; this requires that

$$
\hat{T}\left(z_{a}\right)^{*} \hat{z} \hat{T}\left(z_{a}\right)=\hat{z}+z_{a}
$$

where $\hat{z}=\left(x,-i \hbar \nabla_{x}\right)$ is the quantum operator associated with $z$. One shows that this implies that $\hat{T}\left(z_{a}\right)$ must be the operator

$$
\hat{T}\left(z_{a}\right)=\exp \left(\frac{i}{\hbar} \sigma\left(z_{a}, \hat{z}\right)\right)
$$


whose action on wave functions in the $x$-representation is given by

$$
\hat{T}\left(z_{a}\right) \Psi(x)=\exp \left(\frac{i}{\hbar}\left(p_{a} x-\frac{1}{2} p_{a} x_{a}\right) \Psi\left(x-x_{a}\right) .\right.
$$

Let us interpret Propositions 10 and 12 in terms of the wave functions

$$
\Psi(\check{z})=\exp \left(\frac{i}{\hbar} \varphi(\check{z})\right) \sqrt{\rho}(\check{z})
$$

on $\check{\mathbb{V}}$ introduced in our previous work [8, 17]; $\rho$ is here a de Rham form on $\check{\mathbb{V}}$. Such a wave-function is defined on $\mathbb{V}$, i.e.

$$
\Psi(\gamma \check{z})=\Psi(\check{z}) \text { for all } \gamma \in \pi_{1}(\mathbb{V})
$$

if and only if $\mathbb{V}$ satisfies the $E B K$ condition

$$
\frac{1}{2 \pi \hbar} \oint_{\gamma} p \mathrm{~d} x-\frac{1}{4} m(\gamma) \text { is an integer. }
$$

( $\gamma$ an arbitrary loop on $\mathbb{V}, m(\gamma)$ its Maslov index). We define the action of the Hamiltonian flow $\left(f_{t}^{H}\right)$ on $\Psi$ by

$$
f_{t}^{H} \Psi(\check{z})=\exp \left(\frac{i}{\hbar} \varphi(\check{z}, t)\right) \sqrt{\rho}(\check{z}, t)
$$

where $\varphi(\check{z}, t)$ is the Hamiltonian phase and $\sqrt{\rho}(\check{z}, t)=\sqrt{\rho}\left(f_{t}^{H}(\check{z}), t\right)$. If we now choose for $H$ the translation Hamiltonian $H^{a}(z)=\sigma\left(z, z_{a}\right)$ then in view of (27) the action of $T\left(z_{a}\right)=f_{1}^{H^{a}}$ on $\Psi$ is

$$
T\left(z_{a}\right) \Psi(\check{z})=\exp \left[\frac{i}{\hbar}\left(\varphi(\check{z})+\frac{1}{2} p_{a} x_{a}\right)\right] \sqrt{\rho}\left(T\left(z_{a}\right) \check{z}\right) .
$$

This action of translation operators satisfies, in view of (28), (29) the commutation relations

$$
T\left(z_{b}\right) T\left(z_{a}\right) \Psi(\check{z})=T\left(z_{a}\right) T\left(z_{b}\right) \Psi(\check{z}) .
$$

\section{References}

[1] Abraham, R, Marsden J E Foundations of Mechanics, Second Edition (The Benjamin/Cummings Publishing Company, 1978)

[2] Arnold, V I Mathematical Methods of Classical Mechanics, 2d edition (Graduate Texts in Mathematics, Springer-Verlag, 1978)

[3] BerRy, M V 1984 Quantal phase factors accompanying adiabatic changes, Pro. Roy. Soc. London A 392, 45-57 
[4] DE Gosson, M 1994 On the Leray-Maslov quantization of Lagrangian submanifolds J. Geom. Phys. 13(2), 158-168

[5] De Gosson, M Maslov Classes, Metaplectic Representation and Lagrangian Quantization (Research Notes in Mathematics 95, WileyVCH, Berlin, 1997)

[6] DE Gosson, M 1998 The quantum motion of half-densities and the derivation of Schrödinger's equation, J. Phys., A: Math. Gen. 31, 42394247

[7] De Gosson, M The Principles of Newtonian and Quantum Mechanics; with a foreword by B. Hiley (Imperial College Press, 2001)

[8] DE Gosson, M 2002 The 'symplectic camel principle' and semiclassical mechanics, J. Phys. A: Math. Gen. 35, 6825-51

[9] Gromov, M 1985 Pseudoholomorphic curves in symplectic manifolds, Invent. Math. 82, 307-47

[10] Hofer, H, And Zehnder, E Symplectic Invariants and Hamiltonian Dynamics, Birkhäuser Advanced texts (Basler Lehrbücher, Birkhäuser Verlag, 1994)

[11] Hannay, J H 1985 Angle variable holonomy in adiabatic excursion of an integrable Hamiltonian. J. Phys. A: Math. Gen. 18, 221-30

[12] LERAY, J Lagrangian Analysis and Quantum Mechanics, a mathematical structure related to asymptotic expansions and the Maslov index (the MIT Press, Cambridge, Mass., 1981)

[13] Libermann, P, And Marle, C-M Symplectic Geometry and Analytical Mechanics (D. Reidel Publishing Company, 1987)

[14] Littlejohn, R-G 1986 The semiclassical evolution of wave packets, Physics Reports (Review section of Physics Letters) 138, 4-5, 193-291

[15] Maslov, V P Théorie des Perturbations et Méthodes Asymptotiques (Dunod, Paris, 1972) [original Russian edition: 1965]

[16] Maslov, V P, and Fedoriuk, M V Semi-Classical Approximations in Quantum Mechanics (Reidel, Boston, 1981) 
[17] Montgomery, R 1988 The Connection Whose Holonomy is the Classical Adiabatic Angles of Hannay and Berry and Its Generalization to the Non-Integrable Case, Commun. Math. Phys. 120, 269-294

[18] Talpaert, Y Differential Geometry With Applications to Mathematics and Physics, Pure And Appl. Math. (Marcel Dekker, 2001)

[19] Weinstein, A 1981 Symplectic Geometry, Bull. Amer. Math. Soc. 5, $1-13$

[20] Weinstein, A 1990 Connections of Berry and Hannay Type for Moving Lagrangian Submanifolds, Adv. in Math. 82, 133-59. 